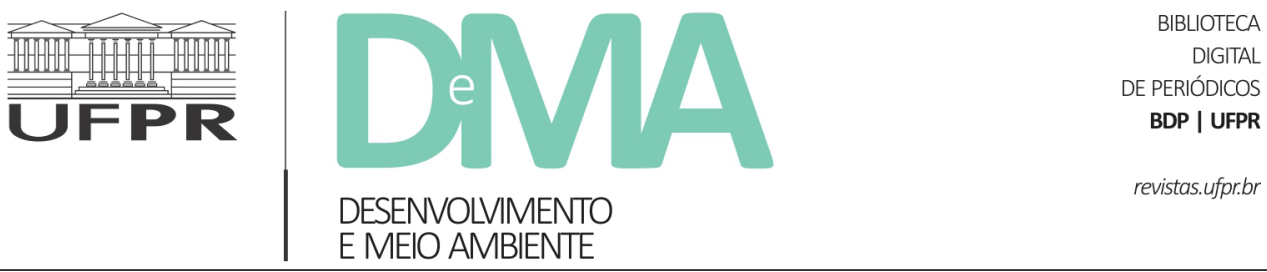

\title{
Implantação de sistemas de dessalinização em comunidades rurais do semiárido do Rio Grande do Norte: percepção social da água potável e das ações voltadas a ampliar o seu acesso universal
}

\section{Installation of desalination systems in rural communities in the semi- arid region of Rio Grande do Norte: social perceptions of drinking water and actions aimed at expanding their universal access}

\author{
Karlia Dalla Santa AMARAL ${ }^{1}$, Nádia D`Paula Santos da Silva MACHADO², Julio Alejandro NAVONI ${ }^{1,3 *}$ \\ ${ }^{1}$ Programa de Pós-graduação em Desenvolvimento e Meio Ambiente, Universidade Federal do Rio Grande do Norte (UFRN), Natal, RN, Brasil. \\ ${ }^{2}$ Secretaria do Meio Ambiente e dos Recursos Hídricos do Rio Grande do Norte, Natal RN, Brasil. \\ ${ }^{3}$ Programa de Pós-graduação em Uso Sustentável de Recursos Naturais, Instituto Federal do Rio Grande do Norte (IFRN), Natal, RN, Brasil. \\ *E-mail de contato: navoni.julio@gmail.com
}

Artigo recebido em 13 de dezembro de 2019, versão final aceita em 29 de julho de 2020, publicado em 25 de novembro de 2020.

RESUMO: Aimplantação de obras de infraestrutura hídrica é uma das linhas de ações voltadas a amparar o desenvolvimento de atividades produtivas no semiárido brasileiro. O presente estudo teve como objetivo avaliar a percepção de comunidades rurais de 24 municípios do semiárido do Rio Grande do Norte, a respeito de tecnologias de dessalinização, voltadas a disponibilizar água para consumo humano. Foram entrevistados, através de três formulários semiestruturados, diferentes grupos de indivíduos de 42 comunidades beneficiadas pelo Programa Água Doce (PAD), dentre estes: gestores dos sistemas de dessalinização $(n=42)$, além das famílias usuárias $(n=160)$ e não usuárias $(n=87)$ da água dessalinizada. De acordo com os resultados encontrados, 53\% das famílias eram usuárias dos sistemas de dessalinização. Gastos mensais fixos como: tarifa de energia elétrica $(62 \%)$, remuneração de operadores $(90 \%)$ e fundo de reserva $(100 \%)$ foram majoritariamente liquidados pela própria comunidade. Apenas $18 \%$ dos usuários consideravam o sistema de abastecimento de água potável adequado antes da instalação dos dessalinizadores. Após a implantação da tecnologia, o modelo de gestão implementado, a performance de serviços e os riscos que acometiam o funcionamento dos sistemas foram pontuados e avaliados. Apesar dos entrevistados reconhecerem a importância dos dessalinizadores na ampliação da segurança hídrica comunitária, o presente trabalho salienta a necessidade de uma maior 
sensibilização da população, quanto aos instrumentos regulatórios e de saúde pública, dirigidos para facilitar a incorporação destas fontes potáveis no semiárido brasileiro.

Palavras-chave: dessalinização; recursos hídricos; comunidades rurais; água potável.

ABSTRACT: Installing water infrastructure is one of the strategies to support the development of productive activities in Brazil's semiarid region. This study seeks to assess how rural community members, from 24 municipalities in the semiarid region of Rio Grande do Norte, perceive desalination technologies aimed at making water available for human consumption. Three semi-structured forms were used to interview different groups of individuals from 42 communities who benefited from the Água Doce Program (PAD), such as managers of desalination systems $(n=42)$, user families $(n=160)$, and non-users $(n=87)$. Results reveal that $53 \%$ of the families were users of desalinization systems. Most of the fixed monthly expenses, such as an electricity tax $(62 \%)$, the operator's salary $(90 \%)$, and a reserve fund $(100 \%)$, were paid by the communities. Only $18 \%$ of users considered that the potable water system was adequate before the desalination plants were installed. After the technology was installed, the management model was implemented, and both service performance and risks affecting how the systems function were scored and evaluated. Although the interviewees recognized the importance of technology in expanding community water security, the present work highlights the need for greater public awareness of regulatory and public health instruments, aimed at facilitating the incorporation of these potable sources in Brazil's semiarid region.

Keywords: desalination; water resources; rural communities; potable water.

\section{Introdução}

No Brasil, o semiárido corresponde a uma área de $10 \%$ do território nacional, concentrando-se a maior parte deste, no Nordeste do país (MMA, 2005). A região Nordeste, é considerada uma das regiões semiáridas mais populosas do planeta, abrigando uma população de 21 milhões de habitantes, dos quais $51,4 \%$ vivem em zona rural (Carvalho \& Egler, 2003; ANA, 2005; Friedel, 2012; IPEA, 2012). Durante os períodos de seca, o abastecimento hídrico das populações rurais dá-se, principalmente, pela água de poços tubulares, já que os recursos hídricos superficiais se encontram indisponíveis ou com uma qualidade imprópria para consumo humano (França \& Moreno, 2017).

Percebe-se que as águas subterrâneas assumem um importante papel frente a cenários de risco hídrico, devido estarem mais protegidas de contaminação, fornecerem água por prolongadas estiagens e encontrarem-se mais próximas do usuário final (Cosme et al., 2011; Hirata \& Conicelli, 2012; Oliveira, 2012; Villar, 2016). No entanto, $70 \%$ da região geológica do semiárido brasileiro é representada pelo embasamento cristalino, o qual caracteriza-se por apresentar pouca disponibilidade hídrica (vazões de $1-3 \mathrm{~m}^{3} / \mathrm{h}$ ), e águas com altos teores de sais (Manoel Filho, 1997; Souza Filho, 1998; Manoel Filho \& Feitosa, 2000; Suassuna, 2002). De acordo com Suassuna (2002), estima-se que $35 \%$ dos 60.000 poços escavados nesse domínio geológico estejam secos, obstruídos ou com teores salinos inadequados ao consumo humano.

Dentre as estratégias utilizadas para a mitigação dos problemas referentes à qualidade de água na região, a instalação de dessalinizadores tem 
sido umas das alternativas empregadas, a fim de atender os usos prioritários da água, conforme as diretrizes da política nacional de recursos hídricos (Brasil, 1997). As tecnologias de dessalinização mundialmente empregadas na atualidade são a de processos térmicos e de separação por membranas (Gharfour et al., 2013). O primeiro tipo é mais utilizado para sistemas de médio e grande porte, enquanto o último, em sistemas de baixa capacidade de produção (Younos \& Tulou, 2005; Karagiannis \& Soldatos, 2008).

Dentre os processos que utilizam pressão hidráulica para separação (microfiltração, ultrafiltração, nanofiltração e osmose reversa), a filtração por osmose reversa foi o tipo de sistema adotado como alternativa mais viável para a extração do excesso de sais, entre outros elementos traços, da água de poços da região semiárida brasileira (Voutchkov, 2004; Santos et al., 2010).

Este tipo de sistema tem se destacado em número de instalações, devido a compactação e resistência do equipamento, além dos baixos custos de instalação e operação, quando utilizado para tratar de baixos a moderados volumes de água (Herold et al., 1998; Porto et al., 2001; Amorim et al., 2004; Soares et al., 2006). No entanto, as estimativas de valores dependem de fatores como a qualidade da água de alimentação e dimensionamento dos sistemas, podendo haver variações para cada localidade. Por exemplo, em sistemas de menores dimensões, alimentados com água salobra, os custos de produção (> $20 \mathrm{~m}^{3} /$ dia) chegam a variar de 5,30 a 12,90 dólares (Karagiannis \& Soldatos, 2008).

Apesar de existirem diferentes tecnologias para tratamento de água, atualmente 1,1 bilhão de pessoas vivem sem acesso a água potável no planeta (Shaw \& Thaitakoo, 2010). A escassez da água potável representa, junto com a falta de saneamento básico, um dos principais riscos à saúde humana, sendo acentuada por problemas globais atuais, como o aquecimento global (IPCC, 2007; Wheeler \& Von Braun, 2013). Apesar da avaliação da vulnerabilidade de sistemas de água potável ser diversa e complexa, há uma unificação dos riscos, no que tange a socialização da natureza e de seus recursos, e das inseguranças introduzidas pela modernização (Beck, 1992; Giddens, 1990; Depla et al., 2020). Deste modo, a gestão da demanda de água é hoje um dos principais problemas na agenda da política de vários países, principalmente para aqueles em desenvolvimento, com muitas áreas periurbanas e rurais, as quais normalmente abrigam populações socialmente excluídas (Franceys \& Gerlach, 2011).

A elucidação das relações intrínsecas entre indivíduos e meio ambiente, bem como dos riscos associados a esta interação, torna-se importante para a compreensão de problemas socioambientais (Larson et al., 2016; Santos et al., 2018). A identificação de grupos populacionais submetidos a situações de risco, bem como a inclusão destes em processos decisórios, é uma tarefa fundamental para a avaliação de exposições diferenciadas e a elaboração de programas preventivos, auxiliando na remedição do processo (Barcellos et al., 1998; Woodhouse \& Muller, 2017). Deste modo, o presente trabalho teve como objetivo avaliar a percepção de comunidades rurais do semiárido do Rio Grande do Norte, a respeito de tecnologias implantadas na região, voltadas a permitir o acesso universal da água, através da implementação dos sistemas de dessalinização. 


\section{Metodologia}

\subsection{Caracterização da área de estudo}

O semiárido brasileiro é uma região definida em lei federal ( $\mathrm{n}^{\circ} 7827$, de 27 de setembro de 1989) e delimitada pelo Ministério da Integração Nacional (MIN, 2005). Engloba o Sudeste e Nordeste do país, sendo que maior parte deste território se concentra na última região $(89,5 \%)$, abrangendo ao todo, nove unidades da Federação. O Rio Grande do Norte (RN) é um dos estados pertencentes a esta região, tendo como limites o Oceano Atlântico (norte a leste) e os estados da Paraíba (sul) e Ceará (oeste). É dividido em 167 municípios, possuindo uma área total de
$52811,126 \mathrm{~km}^{2}$, o que equivale a $3,42 \%$ da área do Nordeste, e a $0,62 \%$ da superfície do Brasil. Encontra-se subdividido em quatro mesorregiões: Oeste Potiguar (62 municípios); Central Potiguar (37 municípios); Agreste Potiguar (43 municípios); e Leste Potiguar (25 municípios) (IBGE, 2017). Do total de municípios, 147 estão inseridos na porção semiárida, representando $92,97 \%$ do seu território (Medeiros et al., 2012; Figueiredo et al., 2016).

O presente estudo abrangeu 24 municípios do RN, distribuídos nas seguintes Mesorregiões: Central Potiguar $(\mathrm{n}=12)$, Agreste Potiguar $(\mathrm{n}=6)$, Oeste Potiguar $(\mathrm{n}=5)$ e Leste Potiguar $(\mathrm{n}=1)$. Dentre os municípios abrangidos, foram amostradas 42 localidades, distribuídas de acordo com a Figura 1.

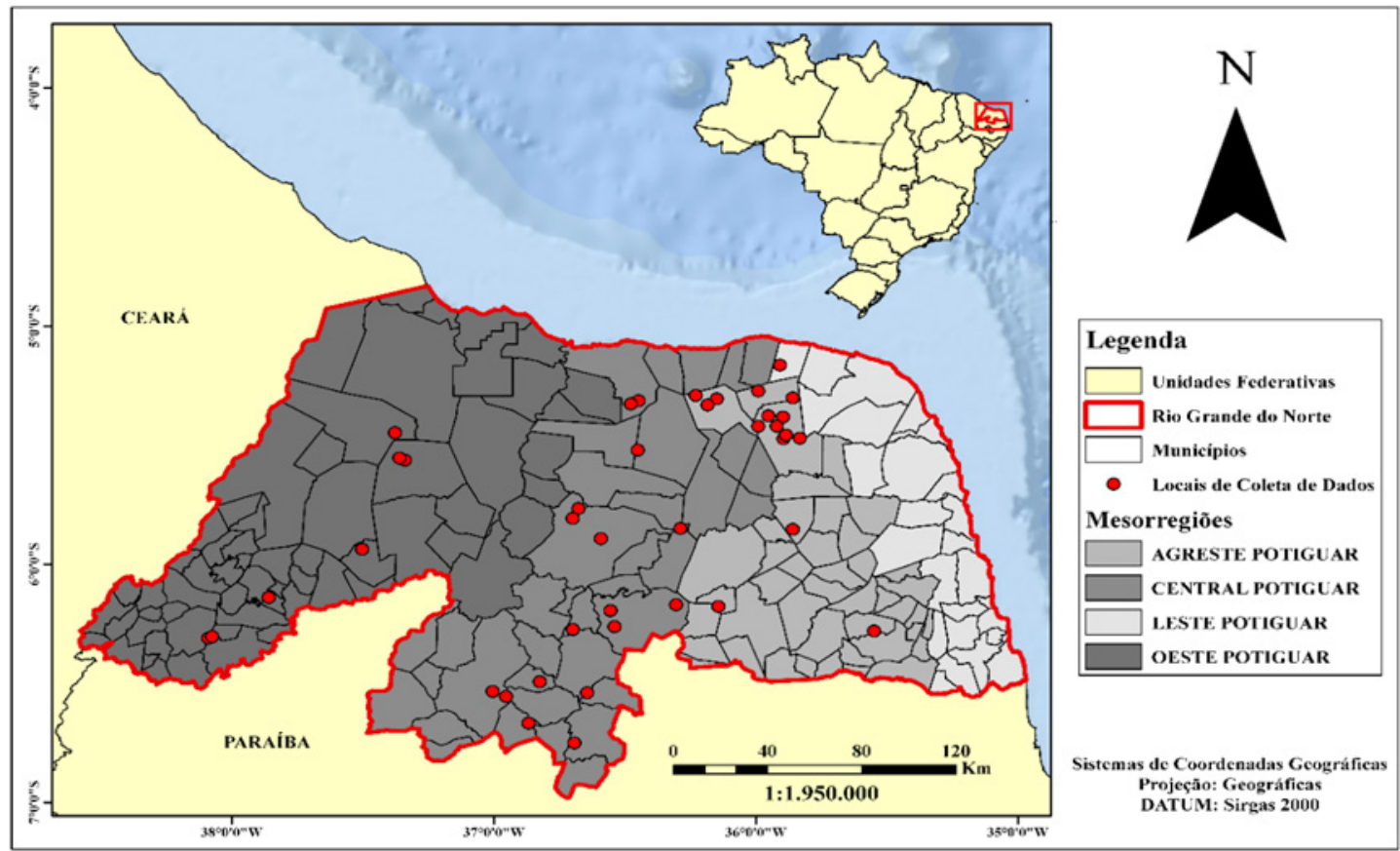

FIGURA 1 - Comunidades amostradas na análise de percepção, Rio Grande do Norte. 


\subsection{Base de dados}

A base de dados utilizada no presente estudo foi disponibilizada pelo Programa Água Doce (PAD), iniciativa governamental, que tem como objetivo garantir a pequenas populações rurais, o acesso a uma água de boa qualidade para consumo humano, através da implantação, recuperação e gestão de sistemas de dessalinização. As informações utilizadas foram coletadas no estado do Rio Grande do Norte (RN), e cedidas pela Secretaria de Meio Ambiente e Recursos Hídricos (SEMARH), órgão responsável pela execução do PAD nesta unidade da Federação. Os dados foram obtidos por meio da aplicação de formulários semiestruturados, em 42 comunidades do RN, entre maio a julho de 2017. As amostragens foram realizadas em localidades que apresentavam sistemas de dessalinização implantados pelo PAD, com no mínimo três meses de funcionamento, a fim de proporcionar uma melhor percepção dos aspectos avaliados.

De acordo com a metodologia do PAD, a hierarquização dos municípios atendidos foi realizada através do Índice de Condição de Acesso à Água, fator calculado segundo as seguintes variáveis: menores índices pluviométricos e de desenvolvimento humano; maiores índices de mortalidade infantil (até 1 ano de idade) e de intensidade de pobreza; e ocorrência de águas subterrâneas salobras (MMA, 2012). Dentre os municípios, foi realizado, ainda, a seleção de comunidades, conforme alguns requisitos como: possuírem no mínimo 20 famílias; poço tubular instalado (vazão média superior a $1000 \mathrm{~L} / \mathrm{h}$ ); água subterrânea com Sólidos Totais Dissolvidos (STD) superior a $1000 \mathrm{mg} / \mathrm{L}$; disponibilidade de energia elétrica e área para implantação do siste- ma. As regras de funcionamento do sistema foram estabelecidas pelo "Acordo de Gestão", documento aprovado e assinado pelas famílias e representantes de instituições públicas, contendo direitos e deveres das pessoas beneficiadas pela água.

\subsection{Estruturação das entrevistas}

A compilação das informações coletadas pelas entrevistas teve como objetivo verificar a percepção de pequenas comunidades rurais, em relação às tecnologias de dessalinização (osmose reversa) implementadas na região, a fim de mitigar a problemática da escassez hídrica local, avaliando pontos positivos e negativos de sua gestão/operação. Ao todo foram aplicados três formulários semiestruturados, sendo o público alvo constituído de gestores locais (operador do dessalinizador, membro do grupo gestor, ou presidente de associação comunitária) e famílias contempladas pelos sistemas de dessalinização implantados pelo PAD. Estas famílias foram classificadas em dois grupos: "Usuárias" $(n=160)$ e "Não usuárias" (n=87), de acordo com a utilização ou não do serviço ofertado.

O primeiro formulário foi aplicado a um gestor $(n=42)$ de cada localidade, a fim de caracterizar os sistemas de dessalinização, através de informações como: frequência semanal de distribuição de água e volume recebido/família; valor de contribuição mensal/família e existência de fundo de reserva comunitário; ente responsável pelo pagamento das taxas de energia elétrica e serviço de operação do sistema; e tipos de usos realizados com a água do rejeito salobro. No segundo formulário, voltado às famílias usuárias, foram entrevistadas 160 famílias, sendo avaliada a percepção deste público em 
relação ao abastecimento de água potável, antes e após a instalação dos sistemas de dessalinização. Estas entrevistas também objetivaram identificar o grau de satisfação dos usuários perante o tipo de gestão implementada pelo PAD e regras estabelecidas (Acordo de Gestão), bem como averiguar sobre os problemas enfrentados pela comunidade. A atuação do PAD junto à comunidade foi avaliada através de dois componentes (mobilização social e sustentabilidade ambiental), responsáveis pela realização de reuniões/oficinas, visando a definição de regras, mediação de conflitos, e orientação para tópicos como saúde e meio ambiente. Em relação ao terceiro formulário, voltado ao público não usuário, foram entrevistadas 87 famílias, verificando o conhecimento destas, a respeito dos sistemas de dessalinização, além dos motivos para o não consumo da água dessalinizada. Os formulários foram compostos, em sua maior parte, por perguntas de resposta fechada, sendo realizada uma amostragem aleatória simples, contemplando $\geq 10 \%$ do total de famílias por localidade. Para o processamento e análise dos dados utilizou-se o software SPSS Statistics (Statistical Package for the Social Science), versão 20 .

\section{Resultados: percepção dos sistemas de dessalinização}

O diagnóstico dos sistemas de dessalinização demostrou que, para o percentual amostrado $(\mathrm{n}=247)$, do total de famílias $(\mathrm{n}=2.391)$ residentes nas localidades contempladas neste estudo, 53\% eram usuárias dos sistemas de dessalinização, enquanto $47 \%$ não usufruíam da benfeitoria.

Os sistemas instalados possuíam diferentes períodos de funcionamento, os quais variaram de 3 a 130 meses. De acordo com análise de correlação de Pearson, constatou-se que as variáveis "tempo de funcionamento dos sistemas" e "quantidade de famílias usuárias" apresentaram uma baixa correlação $(0,19)$, não sendo detectada uma associação relevante entre elas.

A maior parte dos sistemas (76\%) possuía no máximo dois anos de funcionamento, sendo que $19 \%$ possuía de 3 a 4 anos atividade e $2 \%$, um período superior a 10 anos.

A sustentabilidade financeira dos sistemas foi verificada pela análise econométrica, sendo discriminada a titularidade de gastos mensais fixos como: tarifa de energia elétrica, remuneração de operadores e fundo de reserva (recurso destinado a serviços de reparo/manutenção) (Figura 2).

$\mathrm{Na}$ maior parte das localidades, a tarifa de energia elétrica foi liquidada pela própria comunidade (associação comunitária), enquanto que numa menor parte destas, as prefeituras assumiram o compromisso (Figura 2). Os valores pagos, referentes aos serviços de fornecimento de energia (funcionamento do poço e dessalinizador), obtiveram uma variação de até 15 vezes (Tabela 1). Em relação à remuneração do trabalho dos operadores dos sistemas, em $90 \%$ das comunidades o trabalho era voluntário (45\%) ou remunerado pelo público usuário (45\%), enquanto que em apenas quatro comunidades, as prefeituras assumiram a taxa (Figura 2). 


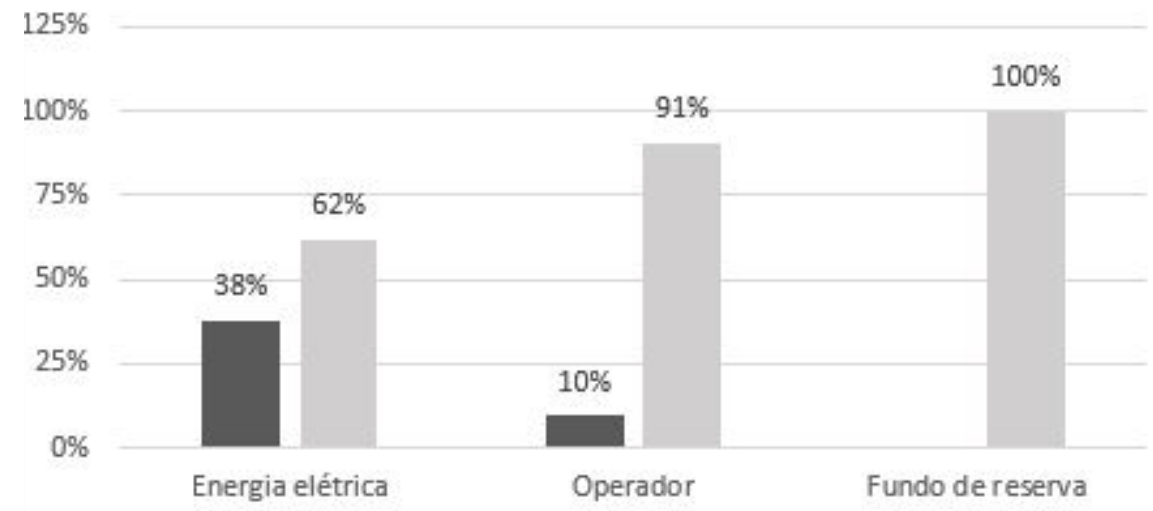

Prefeitura

\section{Comunidade}

FIGURA 2 - Titularidade das despesas de manutenção dos sistemas de dessalinização de 42 comunidades do Rio Grande do Norte.

As gratificações para esta atividade obtiveram variação entre R\$ 3 a R \$ 870 (Tabela 1). Em relação ao fundo de reserva (taxa de contribuição mensal da comunidade para a manutenção dos sistemas), este foi estipulado por localidade, conforme o "Acordo de Gestão Compartilhada", documento firmado entre poder público e a comunidade. De acordo com as entrevistas, foi observado que $73 \%$ das comunidades constituíram um fundo de reserva. A taxa mensal paga por família variou entre $\mathrm{R} \$ 1$ a $\mathrm{R} \$ 5$, com um fundo acumulado, oscilando num intervalo de até 200 vezes (Tabela 1). Em relação à utilização dos produtos (água dessalinizada) e subprodutos (rejeito salobro) do processo de dessalinização, foi verificado que as famílias usuárias consumiam diariamente entre 10 a 120 litros de água dessalinizada, sendo o rejeito salobro utilizado por somente um terço das comunidades (33\%). O subproduto era empregado para fins de dessedentação animal e irrigação, no entanto, a maior parte das comunidades aproveitava esta água apenas para a primeira finalidade (93\%).
Em relação à percepção das famílias usuárias, verificou-se que menos de um quinto destas consideravam o sistema de abastecimento de água adequado ( $9 \%$ excelente e $9 \%$ bom), anteriormente à instalação dos sistemas de dessalinização. Um percentual elevado de entrevistados classificou o serviço como regular (24\%), ruim $(36 \%)$ e péssimo $(22 \%)$, tendo em vista às necessidades da comunidade. Após a implantação dos sistemas, o desempenho de funcionamento foi avaliado mediante modelo de gestão aplicado e performance de serviços efetuados (Figura 3). Nenhum dos itens descritos recebeu avaliação péssima, sendo que uma minoria dos usuários os classificou como regular $(\leq 6 \%)$ e ruim $(\leq 1 \%)$. De maneira geral, as respostas dos entrevistados evidenciaram uma elevada satisfação, com mais de $90 \%$ de respostas satisfatórias (excelente e bom) para cada aspecto e/ou variável avaliada. Entre estes, a "qualidade da água dessalinizada" destacou-se por ser o item com maior percentual de classificação "excelente", 
TABELA 1 - Despesas mensais e receitas de sistemas de dessalinização de 42 comunidades do Rio Grande do Norte.

\begin{tabular}{|c|c|c|c|}
\hline \multicolumn{4}{|c|}{ Despesa mensal (R\$) } \\
\hline & Média & Mínimo & Máximo \\
\hline $\mathrm{N}^{\circ}$ de famílias usuárias & 31 & 7 & 130 \\
\hline Tarifa de energia & 404,2 & 80,00 & $1.200,00$ \\
\hline Serviço de operação do sistema & 190,26 & 3,00 & 870,00 \\
\hline Fundo de reserva & 2,38 & 1,00 & 5,00 \\
\hline Taxa mensal paga por família & 8,6 & 2,00 & 20,00 \\
\hline \multicolumn{4}{|c|}{ Receita (R\$) } \\
\hline \multicolumn{4}{|l|}{ Fundo de Reserva } \\
\hline Saldo acumulado & 985,36 & 50,00 & $10.000,00$ \\
\hline
\end{tabular}

descrita nas palavras dos entrevistados, do seguinte modo: “(...) a água da máquina é muito boa por não ter gosto (...)", “(...) parece com a água mineral comprada (...)". No entanto, uma pequena parte dos entrevistados (4\%) alegou sentir gosto de cloro na água dessalinizada, ou informou que a água permanecia com gosto salobro após o processo de osmose reversa. Quanto ao volume de água disponibilizado por família, considerando sua utilização apenas para consumo humano, a quantidade estipulada conseguiu atender a demanda destinada a esta finalidade, atingindo $94 \%$ de respostas satisfatórias (excelente e bom). O "horário de funcionamento" dos sistemas também se mostrou adequado, sendo que apenas $6 \%$ (regular e ruim) dos entrevistados mencionaram que o período de distribuição de água ocorria matinalmente muito cedo, ou com intervalo de distribuição (horas/dia) muito reduzido, de forma a atender a todas as famílias, representada no seguinte relato: “(...) minha casa é mais distante que as outras e por isso, chego mais tarde, e já tem muita gente para pegar água (...)". Em "serviços de operação dos sistemas", 7\% (regular e ruim) dos entrevistados mencionaram que o sistema poderia ser melhor operado pelo responsável, havendo um menor desperdício da água dessalinizada durante este processo. Em relação as "taxas de contribuição", alguns usuários (4\%) sugeriram tanto um aumento, como uma redução nos valores praticados.

A elevação dos valores foi justificada como alternativa de viabilizar a gratificação dos operadores, nas localidades onde o serviço era realizado de maneira voluntária. Frentes de ação incorporadas ao $\mathrm{PAD}$, envolvendo aspectos relacionados a participação social (componente mobilização social) e ao meio ambiente (componente sustentabilidade ambiental), também foram abordadas neste estudo, mediante a avaliação de sua atuação junto à comunidade, por meio de reuniões e oficinas ministradas (Figura 3). A maior parte dos usuários avaliou as reuniões promovidas pelo primeiro componente como "excelente" e "bom", com $97 \%$ de respostas satisfatórias. No entanto, quando estes entrevistados foram indagados acerca de sua presença nos eventos promovidos, somente $66 \%$ confirmaram participação. Em relação aos eventos promovidos pelo componente "Sustentabilidade ambiental", nenhum dos entrevistados classificou-os negativa- 
mente (regular, ruim e péssimo), obtendo-se $100 \%$ de respostas satisfatórias (excelente e bom). No entanto, menos da metade (38\%) dos usuários afirmaram terem participado das oficinas ministradas, através das quais foram repassadas informações referentes a qualidade da água, a saúde e ao meio ambiente, como representado nos seguintes relatos: “(...) nas reuniões a gente fica sabendo porque o concentrado não pode ser jogado no solo (...)", “(...) as reuniões da sustentabilidade ainda tem pouca gente (...)”, “(...)todo mundo deveria ficar sabendo sobre as doenças que a água não tratada $\operatorname{traz}(\ldots)$..

Para averiguar quais os principais riscos que acometiam o funcionamento dos sistemas, os usuários foram questionados em relação aos problemas que ocorreram durante a sua instalação e operação. Os problemas mencionados foram classificados em cinco categorias: manutenção, gestão, sustentabilidade, obras civis, entre outros. A maior parte das dificuldades foram classificadas como de "manutenção" (74\%), incluindo relatos como: mau funcionamento da bomba do sistema e bomba do poço (bomba queimada ou com baixa potência, implicando em nenhuma ou baixa produção de água), entupimento dos filtros do dessalinizador e vazamentos das caixas d'água. Os problemas classificados como "gestão" (18\%), relacionaram-se á conflitos interpessoais causados por fatores como: não pagamento da conta de energia, dificuldade de relacionamento com o operador do sistema e insatisfação em relação às regras estabelecidas pelo acordo de gestão (quantidade de água distribuída e horário de funcionamento do sistema). Já os referentes a "sustentabilidade" (5\%), foram relativos à insatisfação, quanto a qualidade da água dessali-

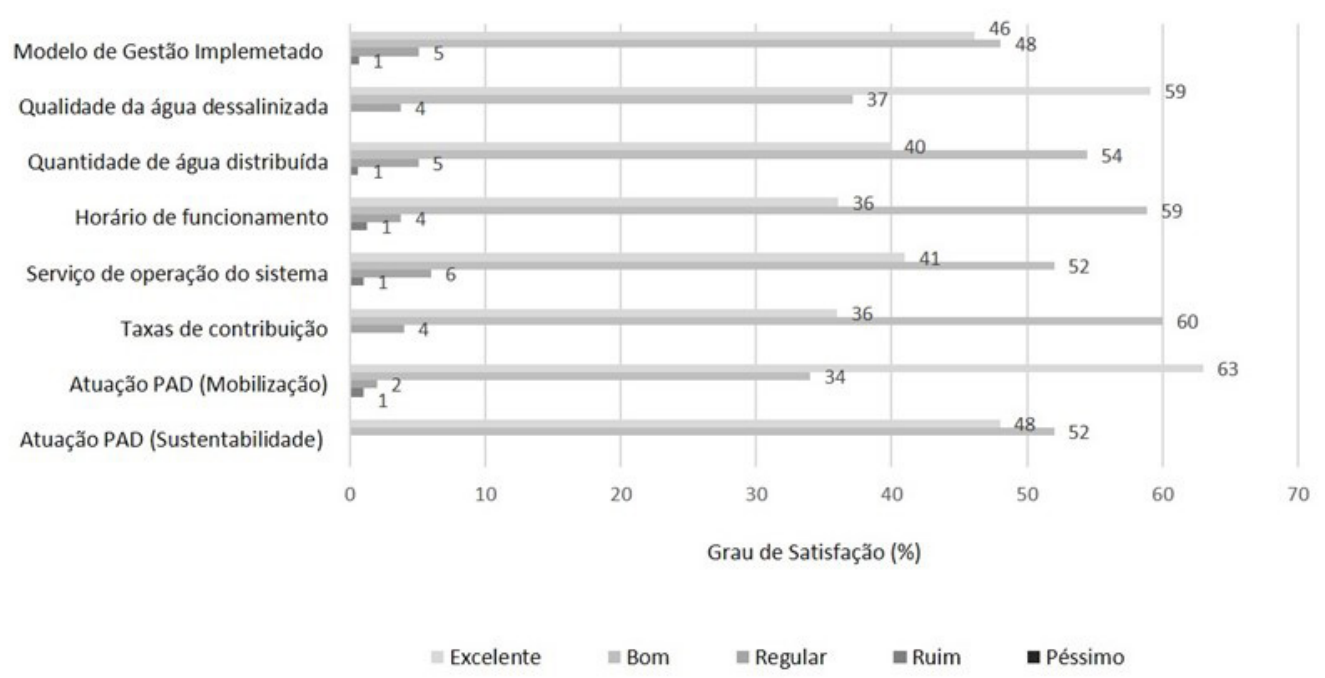

FIGURA 3 - Grau de satisfação dos usuários em relação a gestão/serviços referentes à sistemas de dessalinização implantados pelo Programa Água Doce (PAD), Estado do Rio Grande do Norte. 
nizada. Outras (3\%) dificuldades, corresponderam a situações externas, como oscilação ou falta de energia elétrica. Não foram apontados problemas de "obras civis".

Outro aspecto abordado foi tipo armazenamento da água dessalinizada nas residências, devido este fator possuir uma relação direta com a saúde comunitária. Foi observado que a maior parte dos entrevistados armazenava a água em recipientes com tampa (99\%), o que reflete a assimilação das informações repassadas pelas oficinas promovidas pelo componente sustentabilidade ambiental" (Figura 4). De acordo com as entrevistas, baldes tampados e garrafões de $20 \mathrm{~L}$ foram os utensílios mais frequentemente empregados para o armazenamento da água. Uma menor parcela de entrevistados alegou armazenar água em potes de barro, entre outras formas ${ }^{1}$. Apenas $1 \%$ entrevistados relatou armazenar água em recipientes abertos.

Informações relativas ao público "Não usuário", o qual não consumia a água dessalinizada, foram levantadas através do formulário 3 . Um percentual de $76 \%$ destas pessoas afirmou conhecer o sistema de dessalinização, no entanto, apenas $45 \%$ participaram das reuniões promovidas pelo componente mobilização social, encarregado por

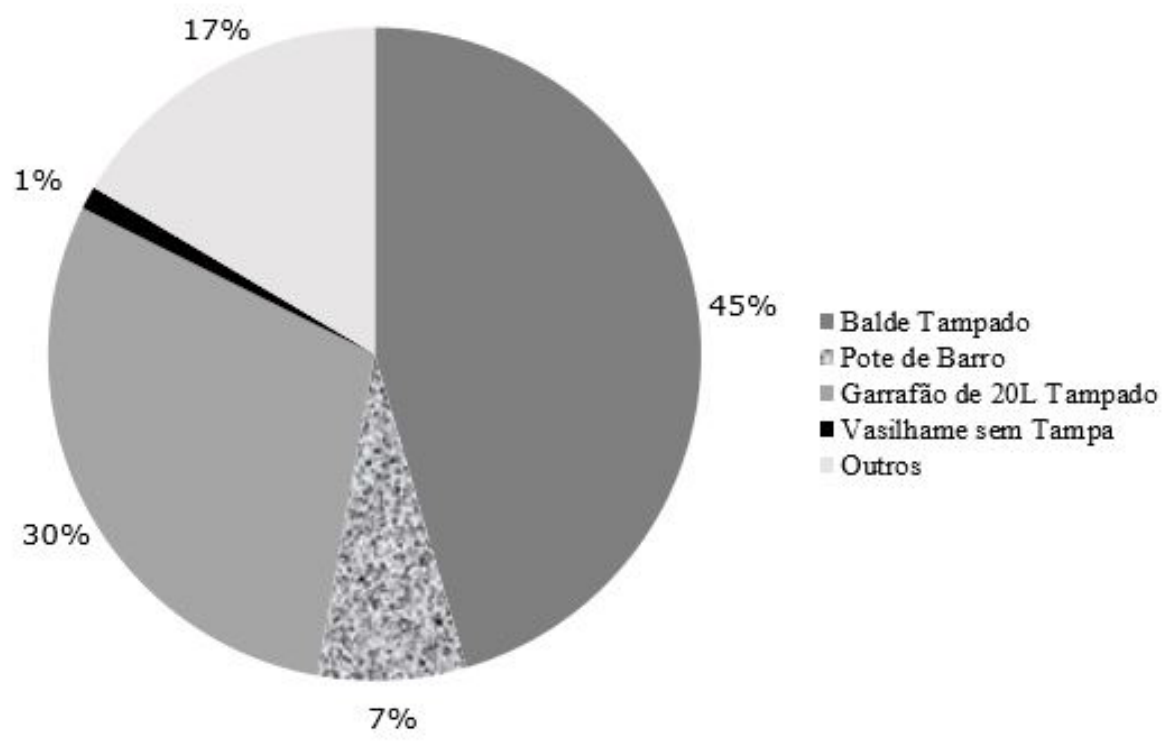

FIGURA 4 - Formas de armazenamento da água dessalinizada nas residências das famílias usuárias.

${ }^{1}$ Formas de armazenamento de água descrita pelos "Usuários" como "Outros": tambores, filtros de barro, caixas d'água, vasilhames e jarras, sendo todos estes recipientes com tampas. 
repassar as orientações gerais de funcionamento dos sistemas. Mais de 50\% dos "Não usuários" não compareceram as reuniões, alegando como motivos: falta de tempo livre por afazeres gerais $(47 \%)$; estar no trabalho (21\%); falta de informação sobre o evento $(17 \%)$; e falta de interesse $(15 \%)$.

Em relação à não utilização da água dessalinizada, a maior parte do público "Não usuário" alegou ser o acesso a outras fontes hídricas (61\%), como a água de cisternas e de carros pipa (28\%), o motivo de tal decisão. Uma menor parcela dos entrevistados (11\%) atribuiu essa escolha a outros fatores ${ }^{2}$. Nenhuma família relatou não ter condições financeiras para contribuir com as taxas de manutenção dos sistemas. Por fim, foi observado que $77 \%$ do público "Não usuário" demostrou interesse em consumir rotineiramente a água dessalinizada, enquanto uma menor parcela deste grupo (23\%) se mostrou totalmente satisfeita com outras fontes de água potável disponíveis.

\section{Discussão}

A maior parte do semiárido brasileiro encontra-se sobre a região geológica do cristalino, apresentando águas subterrâneas com elevados níveis de sais, entre outros elementos químicos (ABAS, 2003). Anteriormente o início da instalação dos sistemas de dessalinização nessa região, as comunidades rurais possuíam como fonte de abastecimento de água potável, a água advinda de cisternas e de carros pipas. Inicialmente, na década de 90 (Programa Água Boa), dessalinizadores foram instalados nas comunidades, sem que elas possuíssem qualquer orientação para sua manutenção a longo prazo, o que findava no sucateamento das máquinas (Oliveira, 2011; MMA, 2012).

Especialistas em percepção de risco, os quais realizaram estudos em diferentes países, demostraram que o fator "confiança" é um dos responsáveis por moldar a aceitação ou rejeição de novas tecnologias implementadas (Feldman, 2012; Ormerod \& Scott, 2013). De acordo com as experiências iniciais das comunidades rurais do semiárido brasileiro, os dessalinizadores eram instalados e funcionavam por um determinado tempo, e ao longo deste período, a água produzida piorava em qualidade, devido à falta de manutenção de filtros e membranas (MMA, 2012). De acordo com alguns estudos, foi verificado que em comunidades que enfrentaram problemas com a qualidade de água, o consumo de água mineral engarrafada era mais regular do que a de fontes locais, o que reforça a ideia de que experiência anteriores influenciam nos hábitos comunitários (Anadu \& Harding, 2000; Butt, 2010).

Neste trabalho, a maior parte dos usuários entrevistados demostrou um alto grau de insatisfação com as fontes de abastecimento de água potável, anteriormente à instalação dos sistemas de dessalinização. A água fornecida por carros pipa, além de ter fornecimento esporádico, por depender de iniciativas governamentais estaduais, também possui uma qualidade diferenciada, por advir de fontes superficiais ricas em matéria orgânica e microrganismos, assim como a água de cisternas (Silans, 2002; Correia et al, 2011). No entanto, verificou-se que após a instalação dos dessalinizadores, quase a metade da população estudada continuou

\footnotetext{
${ }^{2}$ Motivos de não utilização da água dessalinizada descrita pelos "Não usuários" como "Outros": distância percorrida até o dessalinizador, uso de água mineral industrializada, uso de água de poço e falta de recipientes adequados para coleta da água.
} 
consumindo somente a água destas fontes. Um fator que pode ter contribuído para este resultado, foi a maior parte das localidades estudadas (76\%) possuir sistemas instalados há no máximo dois anos, o que representa um curto período de tempo, frente aos efeitos de ações de educativas a longo prazo. Além disso, foi observado um baixo grau de adesão aos eventos promovidos, já que praticamente a metade das famílias entrevistadas não participaram destas ações.

Em relação ao público usuário, verificou-se uma elevada aceitação da tecnologia e modelo de gestão implantados, bem como do produto alvo do processo, a água dessalinizada. O pequeno grau de insatisfação foi devido ao sabor provocado pelos sais ou pelo cloro. As reclamações relativas aos sais foram provenientes de usuários de uma comunidade, onde foi instalado um sistema alimentado por energia solar, o qual encontrava-se em fase de teste. Inicialmente, este sistema não dispunha de bateria, sendo que em dias nublados, a energia gerada não era suficiente para promover um funcionamento contínuo da bomba do dessalinizador, o que contribuía para uma reduzida remoção dos sais. Apesar do nível de sais (Sólidos Totais Dissolvidos) ser superior ao das outras localidades, sua concentração não ultrapassava os limites estabelecidos para consumo humano (1000mg/L) (MS, 2017). Em relação ao Cloro, uma parte dos entrevistados também relataram sentir um forte odor e sabor do produto na água. De acordo com as normas em vigor, uma concentração mínima de $0,2 \mathrm{mg} / 1$ de Cloro deve ser mantida na água, para que esta seja eficientemente desinfectada (MS, 2017). Devido o Cloro evaporar e perder parte de sua ação, quando exposto ao calor, esse produto necessita ser adicionado com determinada frequência a água destinada ao consumo humano (Kitazawa et al., 2006; Puget et al., 2010). No caso da água do dessalinizador, a solução para sua cloração (Cloro granulado 60\%) possuía uma renovação semanal, de acordo com as orientações repassadas aos operadores do sistema nos cursos de capacitação, o que explicaria a percepção dos usuários em relação a este elemento.

Apesar da dessalinização por osmose reversa ser um processo que garante a remoção de sais e de microrganismos da água, a sua qualidade pode ser alterada entre o ponto de produção e o ponto de consumo (Lee, 2013). Normalmente, a falta de limpeza das tubulações e caixas de armazenamento, a cloração inadequada da água e a ausência de treinamento dos operadores, são fatores fortemente relacionados a contaminação do produto final (Abu Amr \& Yassin, 2008; Fragkou \& Mcevoy, 2016; Kumpel \& Nelson, 2016). Além disso, no Brasil, as Soluções Alternativas Coletivas (SACs) de abastecimento de água potável, como os dessalinizadores, são consideradas uma das modalidades menos monitoradas pelo Programa Nacional de Vigilância da Qualidade da Água para Consumo Humano (Vigiagua). De acordo com Raven et al. (1998), a maior parte destes sistemas não são cadastrados no Sistema de Informação de Vigilância da Qualidade da Água para Consumo Humano (Sisagua/Vigiagua), e quando são, em mais da metade destes, não é realizado qualquer tipo de tratamento na água de consumo. Neste trabalho, parte dos usuários detectou a presença de elementos que ocasionam gosto e odor a água, provocando insatisfação em relação a sua qualidade. No entanto, a alteração de algumas propriedades organolépticas da água, nem sempre está associada à sua verdadeira condição de potabilidade. A presença de microrganismos, por exem- 
plo, pode não ocasionar alterações sensorialmente perceptíveis, como cor e turbidez (WHO, 2011).

Em comunidades rurais do semiárido brasileiro, verifica-se que o funcionamento dos sistemas de dessalinização e a qualidade da água gerada dependem, em grande parte, da organização da própria comunidade (Sillans, 2002). Neste trabalho, os custos de operação e manutenção dos sistemas, na maioria das localidades, foi liquidado pelos próprios usuários, o que reflete a capacidade de gestão dos líderes. A maior parte das comunidades conseguiram constituir fundo de reserva, um fator de grande importância, já que a maior parte dos problemas relatados foram referentes a questões de manutenção. Em sistemas com filtração por osmose reversa (vazão de 1.800 litros/hora), o custo das membranas chega a representar $67 \%$ do valor total do aparelho, sendo o item mais caro de manutenção (Campos, 2007). Apesar de algumas das comunidades estudadas não terem constituído fundo de reserva, a troca de membranas dos sistemas estudados (variação de 3, 6 e 9 membranas por sistema) ocorre em média, a cada 6 anos, o que permite um planejamento a longo prazo, para arcar com tal custo de manutenção. No entanto, em comunidades com um número mínimo de usuários, nenhuma das taxas de fundo estipuladas se mostraram adequadas a proporcionar a substituição do ${ }^{\circ}$ mínimo de membranas (3), já que o custo unitário deste item gira em torno de $\mathrm{R} \$ 1.200,00$.

Apesar dos problemas relacionados a gestão serem os segundos mais citados, estes não perfizeram $20 \%$ da totalidade, o que demonstra a importância da incorporação da participação social e de modelos de gestão as tecnologias implementadas. $\mathrm{O}$ fortalecimento da autonomia comunitária pode ser visto como um dos pilares de programas efetivos de combate a riscos, como aqueles relacionados à segurança hídrica. A compreensão dos riscos que acometem a sustentabilidade de ações voltadas a mitigar a problemática da escassez hídrica no semiárido, como a implantação de tecnologias de dessalinização, pode auxiliar numa melhor gestão compartilhada da água (comunidade, entes governamentais e sociedade civil), obtendo-se uma maior efetividade de ações a longo prazo (Marfai et al., 2008).

\section{Conclusão}

A qualidade hídrica reflete diretamente na vida das populações e no seu desenvolvimento. A universalização de fontes potáveis de água na região semiárida é, ainda, uma problemática de política pública atual. Neste trabalho foram detectados uma série de fatores que necessitam ser abordados para maximizar a capacidades da instauração de sistemas de dessalinização em áreas de déficit hídrico, como no semiárido brasileiro. A falta de aderência reportada em algumas ações, demonstrou a necessidade de iniciativas voltadas a garantir a dessalinização como alternativa hídrica sustentável para comunidades rurais de baixa renda. Ações educativas de percepção do recurso hídrico ofertado, que melhore a confiança é um ponto relevante a ser tratado. Percebe-se que a escassez hídrica atua com um fator de vulnerabilidade das comunidades rurais difusas, tornando-as coadjuvantes nas decisões e políticas públicas implementadas na região. Intervenções locais, até mesmo aquelas capazes de gerar melhorias na qualidade de vida das populações, ainda refletem as estruturas históricas de poder nos espaços estudados, e a capacidade social das comunidades em conquistar seus interesses específicos. Além disso, 
estudos futuros dirigidos a avaliar a sustentabilidade hídrica, através dos monitoramentos da qualidade, são fatores diferenciais na optimização e viabilidade do processo de dessalinização, de modo a garantir a qualidade sanitária do produto final.

\section{Agradecimentos}

Os autores agradecem à Coordenação de Aperfeiçoamento de Pessoal de Nível Superior (CAPES) e ao Conselho Nacional de Desenvolvimento Científico $(\mathrm{CNPq})$ pelo apoio financeiro da pesquisa.

\section{Referências}

ABAS - Associação Brasileira de Águas Subterrâneas. Água subterrânea: Minimização das consequências da seca no Nordeste. São Paulo, ABAS Editora, 1. ed., 2003.

Abu Amr, S. S.; Yassin, M. M. Microbial contamination of the drinking water system and its impact on human health in khan Yunis governorate, Gaza strip: seven years of monitoring (2000-2006). Public Health, 22(11), 1275-1283, 2008. doi: 10.1016/j.puhe.2008.02.009

Amorim, M. C. C.; Porto, E. R.; Matos, A. N. B.; Silva, D. F. Diagnóstico de sistemas de dessalinização de água salobra subterrânea em municípios do estado da Paraíba -Brasil. Águas Subterrâneas, 1, 1-17, 2004.

ANA - Agência Nacional de Águas. Panorama da qualidade das águas subterrâneas no Brasil. Brasília, 2005.

Anadu, E.; Harding, A. Risk perception and bottled water use. Journal American Water Works Association, 92(11), 82-92, 2000.

Barcellos, C.; Coutinho, K.; Pina, M. F.; Magalhães, M. M.; Paola, J. C.; Santos, S. M. Inter-relacionamento de dados ambientais e de saúde: análise de risco à saúde aplicada ao abastecimento de água no Rio de Janeiro utilizando Sistemas de Informações Geográficas. Cadernos de Saúde Pública,
14, 597-605, 1998. Disponível em: <https://www.scielosp. org/pdf/csp/1998.v14n3/597-605/pt>

Beck, U. The risk society. Towards a new modernity. Londres: Sage, 1992.

Brasil. Lei n. 9.433, de 8 de janeiro de 1997. Institui a Política Nacional de Recursos Hídricos, cria o Sistema Nacional de Gerenciamento de Recursos Hídricos, regulamenta o inciso XIX do art. 21 da Constituição Federal, e altera $\mathrm{o}$ art. $1^{\circ}$ da Lei $\mathrm{n}^{\circ} 8.001$, de 13 de março de 1990, que modificou a Lei $n^{\circ} 7.990$, de 28 de dezembro de 1989. Brasília: DOU de 9/1/1997.

Butt, K. D. Perceptions of public drinking water in Newfoundland and Labrador: A mixed methods study. St. John's - Canadá, Tese (Doutorado), Memorial University of Newfoundland, 2010.

Campos, R. T. Avaliação benefício-custo de sistemas de dessalinização de água em comunidades rurais cearenses. Revista Economia e Sociologia Rural, 45(4), 963-984, 2007.

Carvalho, O.; Egler, C. A. G. (Orgs.). Alternativas de desenvolvimento para o Nordeste semi-árido. Fortaleza, Banco do Nordeste do Brasil, 2003.

Correia, R. C.; Kiill, L. H. P.; De Moura, M. S. B.; Cunha, T. J. F.; Junior, J.; Araújo, J. L. P. (Orgs.). A região semiárida brasileira. Petrolina, Embrapa Semiárido, 2011. Disponível em: <https://www.alice.cnptia.embrapa.br/handle/ doc/916891>. Acesso em: Jan. 2019.

Cosme, C. R.; Dias, N. D. S.; de Oliveira, A. M.; Oliveira, E. M.; Sousa Neto, O. N. Produção de tomate hidropônico utilizando rejeito da dessalinização na solução nutritiva aplicados em diferentes épocas. Revista Brasileira de Engenharia Agrícola e Ambiental, 15(5), 499-504, 2011.

Delpla, I.; Proulx, F.; Rodríguez, M. J. A methodology to prioritize spatio-temporal monitoring of drinking water quality considering population vulnerability. Journal of environmental management, 255, 109869, 2020. doi:10.1016/j. jenvman.2019.109869

Feldman, D. L. Water. Cambridge, UK, Polity Press., 2012.

Figueiredo, S. C. S.; Júnior, J. C. S.; Figueiredo, J. S. Política de combate dos efeitos da Seca no Semiárido Potiguar: o caso de Riacho do Sangue em Macaíba/RN, 
2002-2010. Caderno de Geografia, 26(45), 201-223, 2016.

Fragkou, M. C.; Mcevoy, J. Trust matters: Why augmenting water supplies via desalination may not overcome perceptual water scarcity. Desalination, 397, 1-8, 2016. doi: 10.1016/j.desal.2016.06.007

Franceys, R. W; Gerlach, E. Consumer involvement in water services regulation. Utilities Policy, 19(2), 61-70, 2011. doi: 10.1016/j.jup.2010.08.003

França, J. M. F.; Moreno, J. C. Uma reflexão sobre os impactos causados pela seca no Rio Grande do Norte de 2012 a 2016. Parcerias Estratégicas, 22(44), 213-232, 2017. Disponível em: <http://seer.cgee.org.br/index.php/ parcerias_estrategicas/article/viewFile/853/781.>

Friedel, M. J. Data-driven modeling of surface temperature anomaly and solar activity trends. Environmental Modelling \& Software, 37, 217-232, 2012. doi: 10.1016/j. envsoft.2012.04.016

Ghaffour, N.; Missimer, T. M.; Amy, G.L. Technical review and evaluation of the economics of water desalination: Current and future challenges for better water supply sustainability. Desalination, 309, 197-207, 2013. doi: 10.1016/j. desal.2012.10.015

Giddens, A. As consequencias da modernidade. Sao Paulo: Editora da UNESP, 1990.

Herold, D.; Horstmann, V.; Neskakis, A; Plettner-Marliani, J.; Piernavieja, G.; Calero, R. Small scale photovoltaic desalination for rural water supply-demonstration plant in Gran Canaria. Renewable Energy, 14(1-4), 293-298, 1998. doi: 10.1016/s0960-1481(98)00080-9

Hirata, R.; Conicelli, B. Groundwater resources in Brazil: a review of possible impacts caused by climate change. Anais da Academia Brasileira de Ciências, 84, 297-312, 2012. doi: 10.1590/S0001-37652012005000037

IBGE - Instituto Brasileiro de Geografia e Estatística. Divisão Regional do Brasil em regiões geograficas imediatas e regiões geofraficas intermediárias 2017. Rio de Janeiro, 2017. Disponível em: <https://biblioteca.ibge.gov.br/visualizacao/livros/liv100600.pdf.>. Acesso em: Set. 2020.

IPCC - Intergovernmental Panel on Climate Change. Impacts, Adaptation and Vulnerability. Working Group II
Contributions to the Fourth Assessment Report of the Intergovernmental Panel on Climate Change, 2007. Disponível em: <https://www.ipcc.ch/site/assets/uploads/2018/02/ ar4-wg2-chapter12-1.pdf $>$. Acesso em: Mar.2019.

IPEA - Instituto de Pesquisas Econômicas Aplicadas. A década inclusiva (2001-2011): desigualdade, pobreza e políticas de renda. Relatórios do IPEA, n. 155. Brasília: IPEA, 2012. <Disponível em: http://repositorio.ipea.gov. br/handle/11058/4639>. Acesso em: Jul. 2019.

Karangiannis, I. C.; Soldatos, P. G. Water desalination cost literature: review and assessment. Desalination, 223, 448456, 2008. doi: 10.1016/j.desal.2007.02.071

Kitazawa, H. Keeping residual chlorine and decreasing unpleasant odor caused by disinfection of tap water. Water Science and Technology: Water Supply, 6(2), 193-199, 2006. doi: 10.2166/ws.2006.069.

Kumpel, E.; Nelson, K. L. Intermittent water supply: prevalence, practice, and microbial water quality. Environmental science \& technology, 50(2), 542-553, 2016. doi: 10.1021/ acs.est.5b03973

Larson, K. L.; Stotts, R.; Wutich, A.; Brewis, A.; White, D. Cross-cultural perceptions of water risks and solutions across select sites. Society \& Natural Resources, 29(9), 1049-1064, 2016. doi: 10.1080/08941920.2015.1122132

Lee, Y. An evaluation of microbial and chemical contamination sources related to the deterioration of tap water quality in the household water supply system. International journal of environmental research and public health, 10(9), 41434160, 2013. doi: 10.3390/ijerph10094143

Manoel Filho, J. Ocorrência das águas subterrâneas. In: Feitosa, A. C.; Manoel Filho, J. Hidrologia: Conceitos e Aplicações. Fortaleza: Serviço Geológico do Brasil CPRM, p.13-33, 1997.

Manoel Filho, J.; Feitosa, F. Hidrogeologia: conceitos e aplicações. Fortaleza: CPRM; Serviço Geológico do Brasil, 2000 .

Marfai, M. A.; King, L.; Sartohadi, J.; Sudrajat, S.; Budiani, S. R.; Yulianto, F. The impact of tidal flooding on a coastal community in Semarang, Indonesia. The Environmentalist, 28(3), 237-248, 2008. doi: 10.1007/s10669-007-9134-4 
Medeiros, S. D. S.; Pinto, T. F.; Hernan Salcedo, I.; Cavalcante, A. D. M. B.; Perez Marin, A. M.; Tinôco, L. B. D. M. Sinopse do censo demográfico para o semiárido brasileiro. Campina Grande: INSA, 2012. Disponível em: <http:/ livroaberto.ibict.br/handle/1/941>. Acesso em: Mar. 2020.

MIN - Ministério da Integração Nacional. Portaria ${ }^{\circ} 89$ de 16 de março de 2005. Brasília: Dou de 21/4/2005.

MMA - Ministério do Meio Ambiente. Panorama da Desertificação do Rio Grande do Norte. Natal: MMA/SERHID, 2005. Disponível em: <http://www.mpgo.mp.br/portalweb/ hp/9/docs/monografia_mma_-_panorama_da_desertificacao_no_rio_grande_do_norte.pdf $>$. Acesso em: 30 jul. 2020 .

MMA - Ministério do Meio Ambiente. Programa Água Doce: Documento Base. Brasília: MMA, 2012. Disponível em: $<$ https://aguadoce.mma.gov.br/anexos/documento-base.pdf>. Acesso em: 15 mai. 2018.

MS - Ministério da Saúde. Portaria 05 de 28 de setembro de 2017. Consolidação das normas sobre as ações e os serviços de saúde do Sistema Único de Saúde. Brasília: DOU de 05/10/2017.

Oliveira, A. M. Potencial de utilização agrícola das águas "salobras" e residuárias da dessalinização por osmose reversa. Mossoró, Dissertação (Mestrado em Ciência do Solo) - UFERSA, 2011.

Oliveira, A. M. P. Mobilização de ions em solos tratados com rejeito da dessalinização por osmose reversa. Mossoró, Dissertação (Mestrado), UFERSA, 2012.

Ormerod, K. J.; Scott, C A. Drinking wastewater: Public trust in potable reuse. Science, Technology, \& Human Values, 38(3), 351-373, 2013. doi:10.1177/0162243912444736

Porto, E. R.; Amorim, M. C. C.; Silva Júnior, L. G. A. Uso do rejeito da dessalinização de água salobra para irrigação da erva-sal (Atriplex nummularia). Revista Brasileira de Engenharia Agrícola e Ambiental, 5(1), 111-114, 2001.

Puget, S.; Beno, N.; Chabanet, C.; Guichard, E.; Thomas-Danguin, T. Tap water consumers differ from non-consumers in chlorine flavor acceptability but not sensitivity. Water research, 44(3), 956-964, 2010. doi: 10.1016/j. watres.2009.10.009.
Raven, P. H.; Berg, L. R.; Johnson, G. B. (Orgs.). Environment. New York, USA, 2nd edition, Saunders College Publishing, 1998.

Santos, R. S. D. S.; Dias, N. D. S.; Sousa Neto, O. N. D.; Gurgel, M. T. Uso do rejeito da dessalinização de água salobra no cultivo da alface (Lactuca sativa $\mathrm{L}$.) em sistema hidropônico NFT. Ciência e Agrotecnologia, 34(4), 983, 2010. Disponível em: <http://www.scielo.br/pdf/cagro/ v34n4/v34n4a26.pdf.>

Santos, M. N. R.; Navoni, J. A.; Amaral, V. S. Avaliação da percepção do risco em uma população do semiárido exposta a tóxicos ambientais. Revista Educação Ambiental em Ação, 62, 2018. Disponível em: <http://www.revistaea.org/artigo. php?idartigo $=3003>$.

Shaw, R.; Thaitakoo, D. (Orgs.). Chapter 1 Water communities: Introduction and overview. In: Shaw, R.; Thaitakoo, D. Water communities. Bingley, Emerald Group Publishing Limited, 2010, p. 1-13.

Sillans, A. P. Alternativas científicas e tecnológicas para abastecimento de água no semi-árido. In: Hofmeister, W. Água e desenvolvimento sustentável no semi-árido. 2002. Fortaleza, Fundação Konrad Adenauer, Séries debates, p. 133-160, 2002. Disponível em: <https://www.kas.de/c/ document_library/get_file?uuid=6868dcf0-2010-3626-b$134-81 \mathrm{fb} 8 \mathrm{~d} 09 \mathrm{fbec} \&$ groupId $=252038>$. Acesso em. Fev. 2020 .

Soares, T. M.; da Silva, I. J.; Duarte, S. N. Destinação de águas residuárias provenientes do processo de dessalinização por osmose. Revista Brasileira de Engenharia Agrícola e Ambiental, 10(3), 730-737, 2006. Disponível em: <www. scielo.br/pdf/rbeaa/v10n3/v10n3a28.pdf.>

Souza Filho, O. A. Geologia e Mapa de Previsão de Ocorrência de Água Subterrânea. Folha SA. 24-YDV Irauçuba, Ceará. Dissertação (Mestrado), UFOP, 1998.

Suassuna, J. Água - um fator limitante para o desenvolvimento do Nordeste? In: Hofmeister, W. Água e desenvolvimento sustentável no semi-árido. Fortaleza, Fundação Konrad Adenauer, Séries debates, p. 117-131, 2002. Disponível em: < https://www.kas.de/c/document_library/ get_file?uuid=6868dcf0-2010-3626-b134-81 fb8d09fbec\&groupId=252038>. Acesso em. Fev. 2020. 
Villar, P. C. As águas subterrâneas e o direito à água em um contexto de crise. Ambiente \& Sociedade, 19(1), 2016. Disponível em: http://www.scielo.br/pdf/asoc/v19n1/pt_ 1809-4422-asoc-19-01-00085.pdf.

Voutchkov, N. Seawater desalination costs cut through power plant co-location. Filtration \& Separation, 41(7), 24-26, 2004. doi: 10.1016/S0015-1882(04)00317-9

Wheeler, T.; Von Braun, J. Climate change impacts on global food security. Science, 341(6145), 508-513, 2013. doi: 10.1126/science. 1239402
WHO - World Health Organization. Guidelines for drinking water quality. 4 Th. Ed. Geneva, 2011. Disponível em: $<$ http://apps.who.int/iris/handle/10665/44584>. Acesso em: Set. 2018.

Woodhouse, P.; Muller, M. Water governance - An historical perspective on current debates. World Development, 92, 225-241, 2017. doi: 10.1016/j.worlddev.2016.11.014

Younos, T.; Tulou, K. E. Overview of desalination techniques. Journal of Contemporary Water Research \& Education, 132, 3-10, 2005. doi: 10.1111?j.1936-704X.205. mp132001002.x 